\title{
PARTICLE-CORE ANALYSIS OF BEAM HALOS IN A SYNCHROTRON
}

M. Ikegami, JAERI, Tokai-mura, Naka-gun, Ibaraki-ken 319-1195, Japan

S. Machida and T. Uesugi * , KEK-Tanashi, Midori-cho, Tanashi-shi, Tokyo 188-8501, Japan

\section{Abstract}

A simple particle-core model for circular accelerators has been constructed assuming that dispersion effects are relatively weak. This model is applicable to a large class of high-intensity rings designed for modest density applications such as spallation neutron sources. Applying this model to isotropic beams in a smooth ring, halo formation processes in the presence of dispersion are investigated. In the analysis, it is found that dispersion matching is essentially important to suppress horizontal beam widening in injection. With dispersion matching, a halo is formed by the particlecore resonance, and its width is a little narrower than that in a straight channel. Dispersion effect on halo formation in high-density situations is also discussed.
\end{abstract}

\section{INTRODUCTION}

Recently, high intensity proton synchrotrons or storage rings have been proposed to be used for various applications such as bunch compression for spallation neutron sources. As uncontrollable beam losses due to halo formation is often considered as a main concern in these machines, increasing attention has recently been given to the beam halo problem in circular accelerators [1]. While the particle-core approach [2] has already been adopted in some of these studies, we propose an alternate particle-core model for beams in circular accelerators in this paper.

In this paper, we consider the simplest situation to clearly bring out the dispersion effect on halo dynamics, namely; we consider a coasting beam circulating in a smooth ring where both the bending and focusing fields are constant (smooth approximation). The emphasis is put upon situations with modest beam density where tune depression, or ratio of depressed tune to undepressed one, is well larger than 0.9 , while halo formation in higher density cases is also discussed.

\section{PARTICLE-CORE MODEL}

The first basis of the particle-core analysis is envelope equations which describe the time-evolution of core envelope. Recently, envelope equations which include effects of dispersion and space charge were derived by Venturini and Reiser [3]. They found that the usual

*Also, University of Tokyo, Hongo, Bunkyo-ku, Tokyo 1130033, Japan horizontal rms emittance $\epsilon_{x}^{2}=\left\langle x^{2}\right\rangle\left\langle p_{x}^{2}\right\rangle-\left\langle x p_{x}\right\rangle^{2}$ is not an invariant of motion in the presence of dispersion. Instead, the generalized emittance $\epsilon_{d x}$ defined as follows is conserved:

$$
\begin{array}{r}
\epsilon_{d x}^{2}=\left(\left\langle x^{2}\right\rangle-D^{2} \Delta^{2}\right)\left(\left\langle p_{x}^{2}\right\rangle-D^{\prime 2} \Delta^{2}\right) \\
-\left(\left\langle x p_{x}\right\rangle-D D^{\prime} \Delta^{2}\right)^{2},
\end{array}
$$

where $\Delta=\sqrt{\left\langle(\delta p / p)^{2}\right\rangle}$ is the rms momentum spread. The independent variable $s$ is the longitudinal distance along the design orbit, and the prime indicates the derivative with respect to $s$. The dispersion function $D$ obeys the equation

$$
D^{\prime \prime}+\left[\kappa_{x}-\frac{K}{2 a(a+b)}\right] D=\frac{1}{\rho},
$$

where $a=\sqrt{\left\langle x^{2}\right\rangle}$ and $b=\sqrt{\left\langle y^{2}\right\rangle}$ are the rms beam widths, and $\rho$ is the average radius of curvature. The generalized perveance $K$ is a measure of the beam density, and $\kappa_{x}$ is a constant which represents the external focusing field strength in the horizontal direction. Note here that $\kappa_{x}$ includes the horizontal focusing effect of the dipole magnets.

The envelope equations with dispersion can be written as

$$
\begin{array}{r}
a^{\prime \prime}+\kappa_{x} a-\frac{K}{2(a+b)}-\frac{\Delta^{2}}{a}\left(\frac{D}{\rho}+D^{\prime 2}\right) \\
-\frac{\epsilon_{d x}^{2}-a^{\prime 2}\left(a^{2}-D^{2} \Delta^{2}\right)+\left(a a^{\prime}-D D^{\prime} \Delta^{2}\right)^{2}}{a\left(a^{2}-D^{2} \Delta^{2}\right)}=0,
\end{array}
$$

and

$$
b^{\prime \prime}+\kappa_{y} b-\frac{K}{2(a+b)}-\frac{\epsilon_{y}^{2}}{b^{3}}=0,
$$

where $\epsilon_{y}$ and $\kappa_{y}$ are, respectively, rms emittance and external focusing field strength in the vertical direction. As easily seen in Eq. (3), the emittance term is modified by adopting the generalized emittance, and the dispersion term is added compared to envelope equations for a straight channel. Simultaneously solving Eq. (2) to Eq. (4), we can obtain the time-evolution of core envelope.

To construct a particle-core model for beams with finite momentum spread, we need to know the matched beam width and coherent tune of these beams. The presence of dispersion causes changes of matched beam size and coherent tune. In the following, we examine these changes due to dispersion considering isotropic situations, namely $\kappa_{x}=\kappa_{y}$ and $\epsilon_{x}=\epsilon_{y}$. 

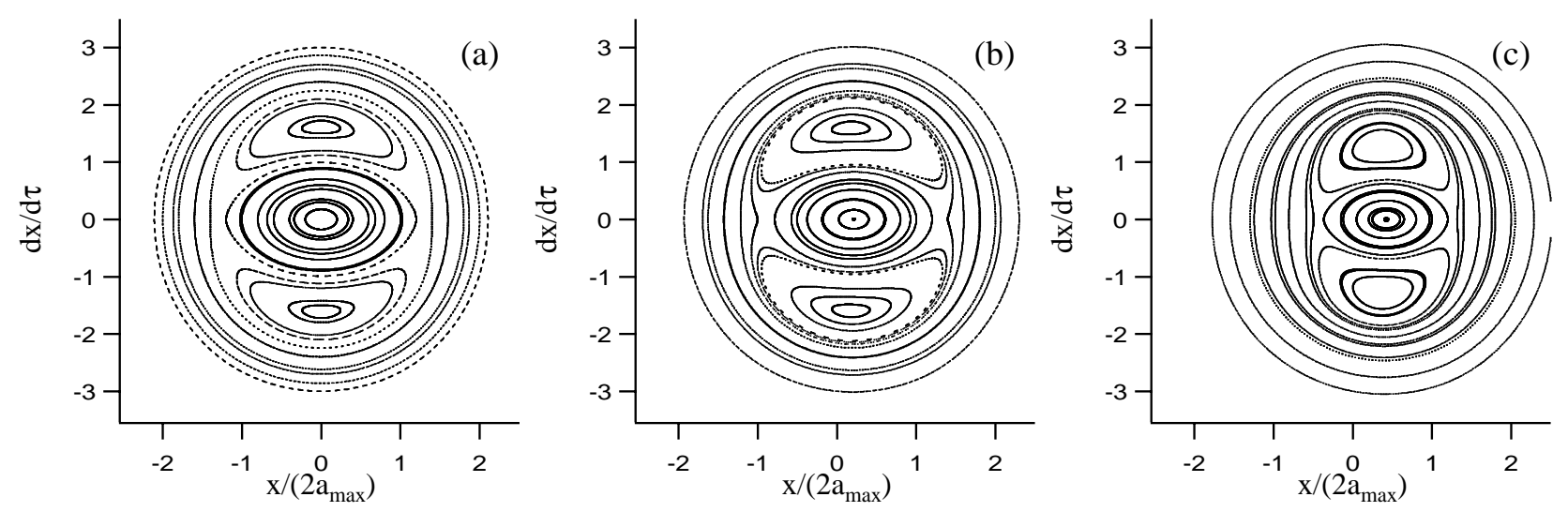

Figure 1: Poincaré plots for test particles in modest density cases with $\eta_{x}=0.9, \mu=1.1$ and $\xi=0.15$. Test particle position scaled by the maximum beam boundary width $2 a_{\max }=2 a(0)$ is taken as the abscissa. Three different $\Lambda$, i.e., (a) $\Lambda=0$, (b) $\Lambda=2$, and (c) $\Lambda=4$ are considered.

Considering situations where dispersion effect is modest, the matched beam widths are obtained [4] as

$$
a_{0}=\hat{a}_{0}\left[1+\frac{\hat{\eta}_{x}^{2}\left(3+5 \hat{\eta}_{x}^{2}\right)}{4\left(1+\hat{\eta}_{x}^{2}\right)\left(1+3 \hat{\eta}_{x}^{2}\right)} \hat{\xi}^{2}\right]
$$

and

$$
b_{0}=\hat{a}_{0}\left[1-\frac{\hat{\eta}_{x}^{2}\left(1-\hat{\eta}_{x}^{2}\right)}{4\left(1+\hat{\eta}_{x}^{2}\right)\left(1+3 \hat{\eta}_{x}^{2}\right)} \hat{\xi}^{2}\right],
$$

where $\hat{a}_{0}$ and $\hat{\eta}_{x}$ are, respectively, the matched beam width and tune depression of the corresponding zero-momentum-spread beam. The parameter $\hat{\xi}=$ $\Delta /\left(\hat{a}_{0} \rho \hat{\eta}_{x}^{2} \kappa_{x}\right)$ is a measure of the strength of dispersion effect. As $\hat{\xi}^{2}$ is typically a few percent in modestdensity circular accelerators [5], fourth or higher order terms of $\hat{\xi}$ are neglected in the derivation of Eq. (5) and Eq. (6).

In mismatched situations, core envelope and dispersion function oscillate around the matched solution. These coherent tunes can be obtained [4] as

$$
\nu_{b}^{2}=\left[2\left(1+\eta_{x}^{2}\right)-\frac{2+14 \eta_{x}^{2}+15 \eta_{x}^{4}-7 \eta_{x}^{6}}{\left(2+\eta_{x}^{2}\right)\left(1+3 \eta_{x}^{2}\right)} \xi^{2}\right] \nu_{x}^{2},
$$

for breathing mode and

$$
\nu_{q}^{2}=\left[1+3 \eta_{x}^{2}-\frac{1+14 \eta_{x}^{2}+30 \eta_{x}^{4}+3 \eta_{x}^{6}}{2\left(1+2 \eta_{x}^{2}\right)\left(1+3 \eta_{x}^{2}\right)} \xi^{2}\right] \nu_{x}^{2},
$$

for quadrupole mode, where $\nu_{x}=\sqrt{\kappa_{x}} \rho$ is the horizontal bare tune. Although one additional mode arises due to coupling with dispersion function, its contribution to envelope oscillation is generally small. The tune depression $\eta_{x}$ is related to $\hat{\eta}_{x}$ as

$$
\eta_{x}^{2}=\left[1+\frac{\left(1-{\hat{\eta_{x}}}^{2}\right)\left(1+2{\hat{\eta_{x}}}^{2}\right)}{\left(1+{\hat{\eta_{x}}}^{2}\right)\left(1+3{\hat{\eta_{x}}}^{2}\right)} \hat{\xi}^{2}\right] \hat{\eta}_{x}^{2} .
$$

The parameter $\xi$ is another measure of the strength of dispersion effect defined by $\xi=\Delta /\left(a_{0} \rho \eta_{x}^{2} \kappa_{x}\right)$. The relation between $\xi$ and $\hat{\xi}$ is easily obtained by using Eq. (5) and Eq. (9).

The second basis of the particle-core analysis is the equation of motions of test particles. Assuming that cores have the KV distribution [6] in the transverse phase space and that test particles have zero angular momentum, equation of motion for test particles initially located on the horizontal plane $\left(y=y^{\prime}=0\right)$ can be written as [7]

$$
x^{\prime \prime}+\kappa_{x} x-\frac{K}{2 a(a+b)} x-\frac{\Lambda \Delta}{\rho}=0,
$$

inside the core $(|x| \leq 2 a)$ and

$$
x^{\prime \prime}+\kappa_{x} x-\frac{K}{x^{2}+|x| \sqrt{x^{2}+4\left(b^{2}-a^{2}\right)}} x-\frac{\Lambda \Delta}{\rho}=0,
$$

outside the core $(|x|>2 a)$, where relative momentum deviation $\Lambda=(\delta p / p) / \Delta$ is a particle-dependent constant. The maximum value of $\Lambda$ typically ranges from two to four, while it is distribution-dependent.

\section{NUMERICAL RESULTS}

Applying the particle-core model described in the preceding section, we examine halo formation in the presence of dispersion. In this section, we concentrate on the cases where breathing mode oscillation is selectively excited. To start with, we consider a modest density beam with $\eta_{x}=0.9$ and $\xi=0.15$. Poincaré surface of section plots for test particles are shown in Fig. 1. In this figure, test particles with three different $\Lambda$ have been considered, while the mismatch factor $\mu=a(0) / a_{0}$ is fixed to 1.1. As readily seen in Fig. 1(a), the width of 2:1 resonance island is a little narrower than that of the corresponding zero-momentum spread beam because of a decrease of coherent tune. As $\Lambda$ is increased, dispersion effect gives a shift of fixed point 

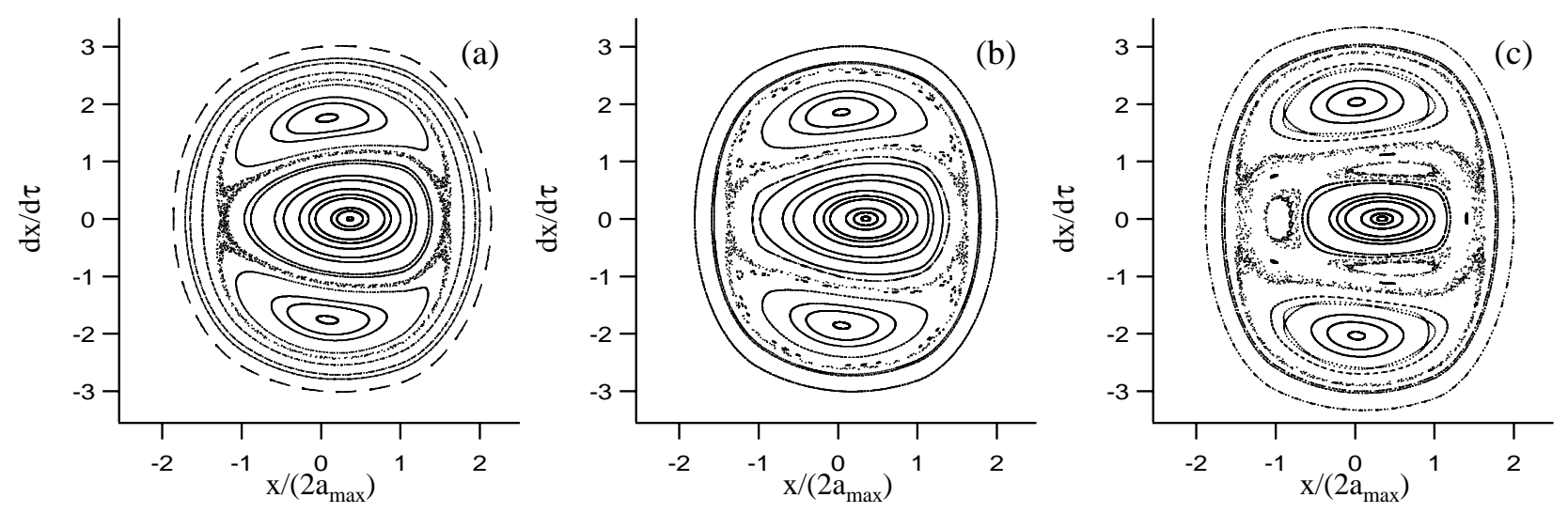

Figure 2: Poincaré plots for test particles in high density cases with $\mu=1.1, \Lambda=4$ and $\xi=0.15$. Beams with three different densities, i.e., (a) $\eta_{x}=0.5$, (b) $\eta_{x}=0.4$, and (c) $\eta_{x}=0.3$ are considered.

locations. At the same time, the width of 2:1 particlecore resonance decreases. As easily seen in Fig. 1, dispersion matching is essential if the maximum value of $\Lambda$ is larger than a certain value, namely; particles with large momentum must be injected into outer side of the core. Without dispersion matching, the shift provides a transport mechanism by which particles initially located inside the core can escape from the core and become halos.

In particle-core analyses for straight channels, only particles which are trapped by the 2:1 particle-core resonance are considered to be halo particles, because particles initially located in the vicinity of the core can not go beyond the separatrix of the resonance. Obviously, this criteria is valid in estimating halo width in the presence of dispersion only in cases where the diffusion due to dispersion mismatch is suppressed. Applying this method, the halo width in the presence of dispersion is found to be a little narrower than that in a straight channel, and typically 1.8 times the maximum core width.

Although this particle-core model is constructed assuming modest density beams, it is applicable to high density cases provided that $\xi$ is small. Poincaré plots for high density beams are shown in Fig. 2, where three different beam densities have been assumed. In increasing beam density keeping $\xi$ to 0.15 , some additional islands of higher order resonances appear around the central stable point. Weak chaosity is observed in all three cases in Fig. 2, which may increase halo intensity.

\section{SUMMARY}

We have constructed a simple particle-core model for beams in circular accelerators, neglecting fourth or higher order contribution of $\xi$. Although this model is efficient only in cases where $\xi$ is small, it is applicable to a large class of circular accelerators designed for modest-density applications and it provides us useful information on halo dynamics in these machines. Especially, this model is sufficient for halo studies in most rings designed as a bunch compressor for spallation neutron sources.

\section{REFERENCES}

[1] S. Machida and M. Ikegami, in Workshop on Space Charge Physics in High Intensity Hadron Rings, Edited by A. U. Luccio and W. T. Weng, AIP Conference Proceedings No. 448, (AIP, New York, 1998), p. 73; J. A. Holmes, J. D. Galambos, D. K. Olsen, and S. Y. Lee, ibid., p.254; in Proceedings of the Sixth European Particle Accelerator Conference, (IOP, London, 1998), p. 279; J. A. Holmes, J. D. Galambos, D. K. Olsen, J. H. Whealton, M. Blaskiewicz, A. Luccio, and J. BeebeWang, ibid., p. 1109.

[2] T. P. Wangler, K. R. Crandall, R. Ryne, and T. S. Wang, Phys. Rev. ST-AB 1, 084201 (1998), and references therein.

[3] We have adopted envelope equations derived in, M. Venturini and M. Reiser, Phy. Rev. Lett. 81, 96 (1998), as the starting point of our analysis, while an alternative form of envelope equations is derived in, S. Y. Lee and H. Okamoto, Phy. Rev. Lett. 80, 5133 (1998).

[4] M. Ikegami, S. Machida, and T. Uesugi, in preparation.

[5] For example, $\xi=0.15$ for the proton storage ring proposed as an option in Nutron Science Project at JAERI. See for detailed design parameters of this ring, M. Kinsho, J. Kusano, M. Mizumoto, and F. Noda, in Proceedings of the Sixth European Particle Accelerator Conference, (IOP, London, 1998), p. 844.

[6] I. M. Kapchinskij and V. V. Vladimirskij, in Proceedings of International Conference for High Energy Accelerators, (CERN, Geneva, 1959), p. 274.

[7] See for example, O. D. Kellog, Foundation of Potential Theory, Frederick Ungar Publishing Company, New York, 1953, Chap. IV. 